\title{
La inflación en méxico y zacatecas, período 2012-2017
}

\section{Inflation in mexico and zacatecas, period 2012-2017}

DOI: $10.46932 /$ sfjdv3n1-058

Received in: Jan 30st, 2021

Accepted in: Feb 1th, 2022

\section{Saúl Robles Soto}

Doctor en Ciencias Económicas, Docente-Investigador de la Unidad Académica de Economía de la Universidad Autónoma de Zacatecas, México,

E-mail: saul.robles@ymail.com

\section{Arturo Martínez Macías}

Doctor en Ciencia Política, Docente-Investigador de la Unidad Académica de Economía de la Universidad Autónoma de Zacatecas, México.

\section{Claudia Esther Aguilar Torres}

Doctora en Ciencia Política, Docente-Investigadora de la Unidad Académica de Economía de la Universidad Autónoma de Zacatecas, México.

E-mail: clauaguila@yahoo.com

\section{Elena Anatolieva Zhizhko}

Doctora en docencia Superior, docente-imvestigadorea de la unidad Académica de docencia superior de la Universidad Autónoma de Zacatecas, México,

E-mail: eanatoli@yahoo.com

\section{Gonzalo Llamas Bernal}

Maestro en Economía, Docente-Investigador de la Unidad Académica de Economía de la Universidad

Autónoma de Zacatecas, México,

E-mail: gonzalo.llamas@yahoo.com.mx

\section{RESUMEN}

El presente trabajo de investigación es resultado de un rastreo contante de la variable inflación tanto en México como en Zacatecas por parte de cinco docentes-investigadores de dos unidades académicas de la Universidad Autónoma de Zacatecas, México durante el periodo 2012-2017. Los datos que se manejan fueron obtenidos de fuentes directas e indirectas que al contrastarlas con las oficiales (tanto federales como estatales), nos indican que la inflación ha tenido repercusiones directas en las compras de bienes y servicios que realizan de manera periódica la clase trabajadora de México y Zacatecas. En el sistema económico mexicano, la inflación en el período 2012-2017 se mantuvo bajo control por parte de las autoridades encargadas de ello. Es uno de los mayores logros obtenidos en materia económica del actual sexenio, una inflación por debajo del 10\%. Ese indicador económico en México es resultado de una férrea contención del salario de la mayoría de los trabajadores, los cuales sufren el embate empresarial y gubernamental, lo mismo que de sus mismos sindicatos al no propiciar acciones tendientes a subir el bajo salario que tienen desde hace tiempo. Esta tendencia de bajas salariales en el sistema mexicano es resultado de negociaciones anuales sostenidas por la llamada comisión tripartita: trabajadores representados por sus sindicatos, empresarios que se agrupan en varias organizaciones y el sector gubernamental encabezado por la Secretaria de Trabajo y Previsión Social. Al mismo tiempo se 
escuchaban voces del mismo sector empresarial que proponían de inmediato un aumento a los salarios mínimos en México, argumentando que debe ser por arriba de los 150 pesos al día. También era noticia la posición de sectores económicos canadienses que establecen una posición de crítica contante hacia el bajo nivel salarial de la mayoría de los trabajadores en México.

Palabras claves: inflación, economía, México, Zacatecas.

\begin{abstract}
The present research work is the result of a continuous tracking of the inflation variable in both Mexico and Zacatecas by five teacher-researchers from two academic units of the Autonomous University of Zacatecas, Mexico during the period 2012-2017. The data handled were obtained from direct and indirect sources that when contrasted with the official ones (both federal and state), indicate to us that inflation has had direct repercussions on the purchases of goods and services made periodically by the working class in Mexico and Zacatecas. In the Mexican economic system, inflation in the 2012-2017 period was kept under control by the authorities in charge of it. It is one of the greatest achievements obtained in economic matters of the current six-year term, an inflation below 10\%. This economic indicator in Mexico is the result of a strong containment of the salary of most workers, who suffer the onslaught of business and government, as well as their own unions by not promoting actions aimed at raising the low salary they have had for some time. This trend of low salaries in the Mexican system is the result of annual negotiations held by the so-called tripartite commission: workers represented by their unions, employers grouped in various organizations and the government sector headed by the Secretary of Labor and Social Welfare. At the same time, voices from the same business sector were heard proposing an immediate increase in Mexico's minimum wages, arguing that they should be above 150 pesos per day. Also in the news was the position of the Canadian economic sectors, which established a position of constant criticism towards the low salary level of the majority of workers in Mexico.
\end{abstract}

Keywords: inflation, economy, Mexico, Zacatecas.

\title{
1 INTRODUCCIÓN
}

La inflación es un incremento en los precios de los bienes y servicios de acuerdo a cierto período de tiempo, es útil en economía para ver la capacidad del poder adquisitivo de las personas.

Un 75\% de los trabajadores en México han padecido repercusiones negativas en su capacidad de compra y salen afectados por los índices inflacionarios de los últimos siete años.

De acuerdo a cifras oficiales, la inflación en México para el mes de septiembre del 2017 es del $7.2 \%{ }^{1}$, lo que no contrasta mucho con el indicador oficial que es del 6.6\% (una diferencia de apenas $0.6 \%$ ).

\footnotetext{
${ }^{1}$ Un cálculo que realizamos en el Laboratorio de Economía Aplicada (LEA) de la Unidad Académica de Economía de la UAZ desde hace 9 años de manera ininterrumpida.
} 


\subsection{INDICADORES DE LA INFLACIÓN EN MÉXICO, 2012-2017}

En base a la siguiente tabla número 1, se tienen los índices inflacionarios del período 2012-2017 en México, los cuales desde el año 2012 se tuvo un promedio de 3.57\%, creciendo hasta el año 2014 con el $4.08 \%$.

Posteriormente en el año 2015 se tiene una disminución notable hasta alcanzar el $2.13 \%$, aunque vale decir que los siguientes años la inflación ha subido hasta alcanzar los niveles de $3.36 \%$ para el año 2016 y para el año de 2017 hasta el mes de octubre el índice inflacionario había alcanzado el 6.66\%, con visos de seguir aumentado dadas las condiciones de fin de año, en las cuales los precios de los bienes y servicios tienen un repunte por el aumento del nivel monetario en el país.

Tabla número 1, inflación anual en México, período 2012-20
\begin{tabular}{|l|l|}
\hline años & Inflación en \% anual \\
\hline 2012 & 3.57 \\
\hline 2013 & 3.97 \\
\hline 2014 & 4.08 \\
\hline 2015 & 2.13 \\
\hline 2016 & 3.36 \\
\hline 2017 & 6.66 \\
\hline
\end{tabular}

Fuente: elaboración propia, LEA, Inegi, Banco de México y periódico La Jornada, sección dinero, 8 de septiembre del 2017.

Como se desprende de manera inmediata del cuadro número 1, la principal institución para el control de la inflación que es el Banco de México, de acuerdo a la Constitución Política de los Estados Unidos Mexicanos.

Las principales causas de los efectos inflacionarios para el gobierno federal son:

1.- Gasolinazos, que no se pudieron detener, y más aún subieron de manera constante los precios con la escasez de combustibles traídos del Sur de Texas y de la Florida, cuyas instalaciones han sido afectadas por ciertos fenómenos naturales.

La cantidad de combustibles que se importó de los citados estados por parte del gobierno federal vía Pemex es del 60\% del consumo nacional en el mes de septiembre del $2017 .^{2}$

2.- La devaluación del peso con respecto al dólar afectó de sobremanera a las familias y empresas que dependen de dicha moneda. Las familias porque reduce su gasto diario por los recursos que reciben de los connacionales y que suman más de 16 mil millones de dólares hasta el mes de septiembre del 2017.

Asimismo, las empresas que tienen deudas en dólares se ven afectadas de manera muy directa al tener que pagar intereses que no estaban contemplados del todo en sus presupuestos con la subida del dólar con respecto al peso mexicano.

El dólar para el mes de septiembre del 2017se ubica por arriba de los 18 pesos y subiendo.

\footnotetext{
${ }^{2}$ Información contenida en el LEA, UAEUAZ, septiembre del 2017.
} 
De esta manera, el indicador inflacionario para el mes de agosto fue del $0.50 \%$ en comparación al mes de julio del 2017.

Hasta el mes de septiembre del 2017, la inflación anual para México se ubicó en 6.66\%, el registro más alto desde el año 2001, cuando lo gobernaba el panista Vicente Fox.

Asimismo, el gobierno peñista al final del año 2012 y todo el 2013 tuvo una inflación del 3.77\%, muy baja con el $6.66 \%$ que tiene en el año 2017 para el mes de septiembre.

\subsection{ES UNA DE LAS ENORMES DEUDAS PENDIENTES QUE EL GOBIERNO DE PEÑA NIETO NO PUEDE OCULTAR.}

Cuáles fueron los productos y servicios que incrementaron más sus precios, aumentando con ello de manera notable el nivel inflacionario en México? gas LP, fresas, plátano, jitomate, cebolla y aguacate, en los servicios, entradas al cine, el cobro en las colegiaturas de escuelas privadas (muchas ya oficiales), así como el precio del pasaje urbano y suburbano.

Es notoria la disminución del poder adquisitivo de la población al tener aumentos significativos para el año 2017 en los índices inflacionarios para el sistema económico mexicano, al grado de empezar a tocar alarmas en las autoridades financieras y hacendarias del país.

Máxime que el gobernador del Bando de México dejó el puesto a fines del mes de noviembre del 2017 y el sucesor no tendría el respaldo correspondiente del Dr. Agustín Cartsens el cual sorteó una serie de dificultades económicas en el sistema sin que la inflación suba más allá del $10 \%$.

Se debe hacer notar que el actual modelo económico implementado en México (neoliberal), ha resultado muy dañino para las capas poblacionales más desprotegidas del país, en contraposición a ello, ha beneficiado a un segmento muy reducido de la población, al grado de tener dentro de ellos entre los más ricos del mundo para el año 2017.

Realizando un comparativo de las cifras anuales de la inflación, se tiene la siguiente gráfica número 1 que da cuenta de ello. 
Gráfica número 1, Inflación anual en \% para México, período 2012-2017.

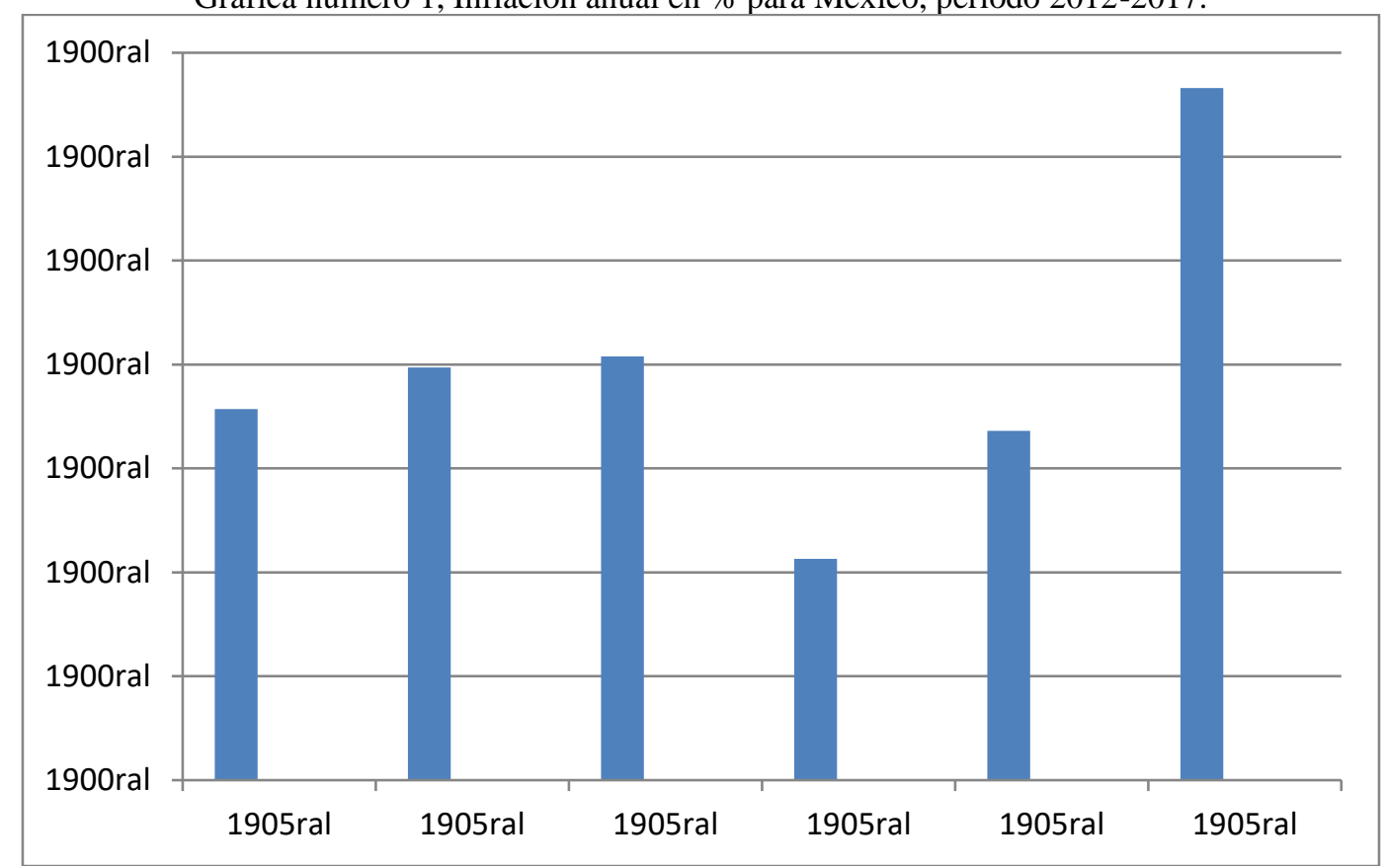

Fuente: Información contenida en el LEA, UAEUAZ, septiembre del 2017.

Como se observa la gráfica, la inflación tuvo una disminución considerable en el año 2015, que quiso ser la más baja del sexenio, para apoyar en cierta medida las impopulares reformas estructurales de EPN, sin embargo, fue una quimera que no hizo verano porque para los siguiente años la inflación se ha incrementado a niveles alarmantes.

\subsection{LA INFLACIÓN EN EL ESTADO DE ZACATECAS, 2012-2017}

En el estado de Zacatecas la inflación se ha manifestado de manera desigual que la referida en el resto del país. Quizás por la movilidad de trabajadores hacia Norteamérica que repercute de manera directa en ciertas zonas del estado (municipios expulsores por tradición de trabajadores hacia USA e inclusive hacia los estados circunvecinos).

Es necesario resaltar que el estado de Zacatecas es una de las entidades federativas de México que ha sufrido un estancamiento económico en varias décadas entre otras cosas por la falta de visión de sus gobernantes que le han apostado a un modelo económico alejado de la realidad, esto es una incipiente industrialización con llegad en masa de empresas asiáticas que más que beneficiar realmente al estado lo han orillado a una dependencia cada vez mayor con respecto a la federación. ${ }^{3}$

Así, la inflación repuntó por arriba de la media nacional, los datos que tenemos registrados en los años del 2012 al 2017 son los siguientes contenidos en la tabla número 2.

\footnotetext{
${ }^{3}$ El estado de Zacatecas recibe un presupuesto anual de la federación por un 95\%, quedando solamente el 5\% de recursos propios del estado, una proporción muy desigual que origina un atraso secular para el estado por décadas.
} 
Gráfica número 2, inflación anual en el estado de Zacatecas en \%, período 2012-2017

\begin{tabular}{|l|l|}
\hline años & Inflación anual en \% \\
\hline 2012 & 4.01 \\
\hline 2013 & 4.47 \\
\hline 2014 & 4.58 \\
\hline 2015 & 3.10 \\
\hline 2016 & 3.86 \\
\hline 2017 & 7.16 \\
\hline
\end{tabular}

Fuente: Información contenida en el LEA, UAEUAZ, septiembre del 2017.

¿Por qué se tiene una inflación por arriba de lo nacional? Porque en el estado de Zacatecas se calcula el índice inflacionario en base a la ciudad de Fresnillo, la cual en los últimos años se ha caracterizado por ser el centro de diversos actos delicuenciales que han orillado de manera directa e indirecta que suban los precios de los bienes y los servicios en mayor magnitud que en el resto del país.

Para el INEGI en sus indicadores acerca de la inflación tiene como referente la ciudad de Fresnillo que si bien es la ciudad más poblada del estado, también es un referente obligado del trasiego de droga por estar ubicado en una zona estratégica en el país (es un paso obligado para el norte de México).

Ello ha ocasionado una inflación más allá de la media nacional, lo cual afecta de sobremanera a los habitantes del entorno, los cuales deben estirar su magro salario que reciben con respecto a los aumentos generalizados de los bienes y servicios, sobre todo los más elementales para subsistir como son alimento, vestido y vivienda, amén de los incrementos generalizados en el transporte urbano y semiurbano.

Los datos contenidos en la tabla número 2 nos indican el índice inflacionario anual en el estado de Zacatecas para el período 2012-2017, información que de manera inmediata refleja un aumento en los precios de los bienes y servicios más allá de los reflejados en el promedio nacional como nos especificaba la tabla número 1 en páginas anteriores.

En la gráfica número 2 se presenta el índice inflacionario anual para el estado de Zacatecas en el período 2012-2017, de inmediato se refleja el aumento en el año 2017 por arriba del 7\%, un dato que representa una inflación por arriba de la media nacional que hasta octubre del 2017 estaba en el 6.6\%.

Este aumento es muy notorio, en la medida que se puede reflejar con mayor intensidad en cuanto a crecimiento en los próximos años, máxime que el presidente Enrique Peña Nieto dejará el poder en el mes de diciembre del año 2018.

Recordemos que un presidente en México que no controle la inflación afectará de sobremanera al partido político en turno, como es el caso de Enrique Peña Nieto.

Esta variable económica (inflación), al afectar a los bolsillos de los mexicanos, dejará una huella difícil de borrar para las próximas generaciones, por lo que el gobierno federal deberá tener mucho cuidado en su manejo. 
Gráfica número 2, índice inflacionario anual en el estado de Zacatecas para el período 2012-2017.

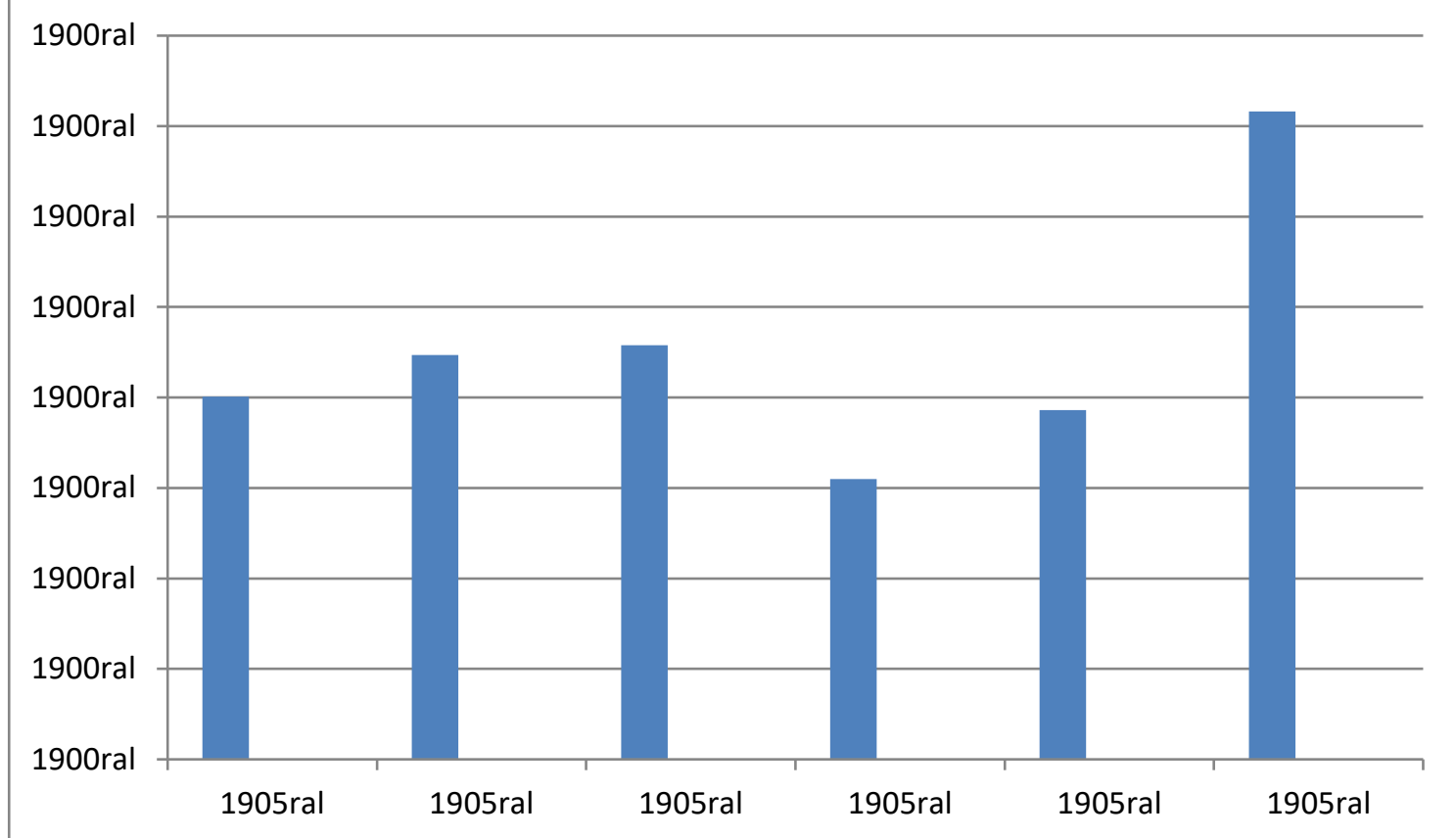

Fuente: Información contenida en el LEA, UAEUAZ, septiembre del 2017.

Fresnillo ciudad está catalogada en el indicador de INEGI como la ciudad número 10 como la ciudad más cara de México.

Asimismo, el estado de Zacatecas es gran propulsor de trabajadores a USA y Canadá, amén de otras ciudades del interior de México como Monterrey, Guadalajara, Aguascalientes, San Luis Potosí y León por nombrar algunas de las más cercanas.

De acuerdo a INEGI, existen en Zacatecas 14 mil desempleados para el mes de septiembre del 2017, que tienen las capacidades para trabajar pero no existen las empresas para ocuparlos, de ahí que emigren hacia otras partes. El gobernador en su informe argumentó que se han creado 8 mil nuevos empleos fijos y duraderos, lo que refleja de inmediato un déficit marcado de 6 mil plazas que no se tienen en el estado zacatecano.

Realizando un comparativo entre el estado de Zacatecas y México, se advierte de inmediato la proporción de la inflación anual entre uno y otro, destacándose un mayor índice inflacionario para el estado con respecto al nacional.

Mientras que para México la inflación en el año 2012 era de 3.7\%, en el estado de Zacatecas la cifra llegaba al 4\%, en el siguiente año, la inflación en México se ubicaba en el 3.85 y en el estado de Zacatecas estaba por el orden del 4.3\%, en iguales circunstancias están los años 2014, 2016 y 2017.

Debemos resaltar que la inflación en el período analizado solamente ha disminuido en el año 2015, quizás por haber sido el año en el cual se plasmaron los acuerdos cupulares de los principales partidos 
políticos mexicanos (PRI, PAN y PRD) en el llamado acuerdo por México, que palió el proceso inflacionario.

Sin embargo, para el año 2016 la inflación tanto en México como en Zacatecas volvió a subir hasta llegar en el año 2017 a niveles históricos, Zacatecas por arriba del 7\% y México llegando al 6.6\%.

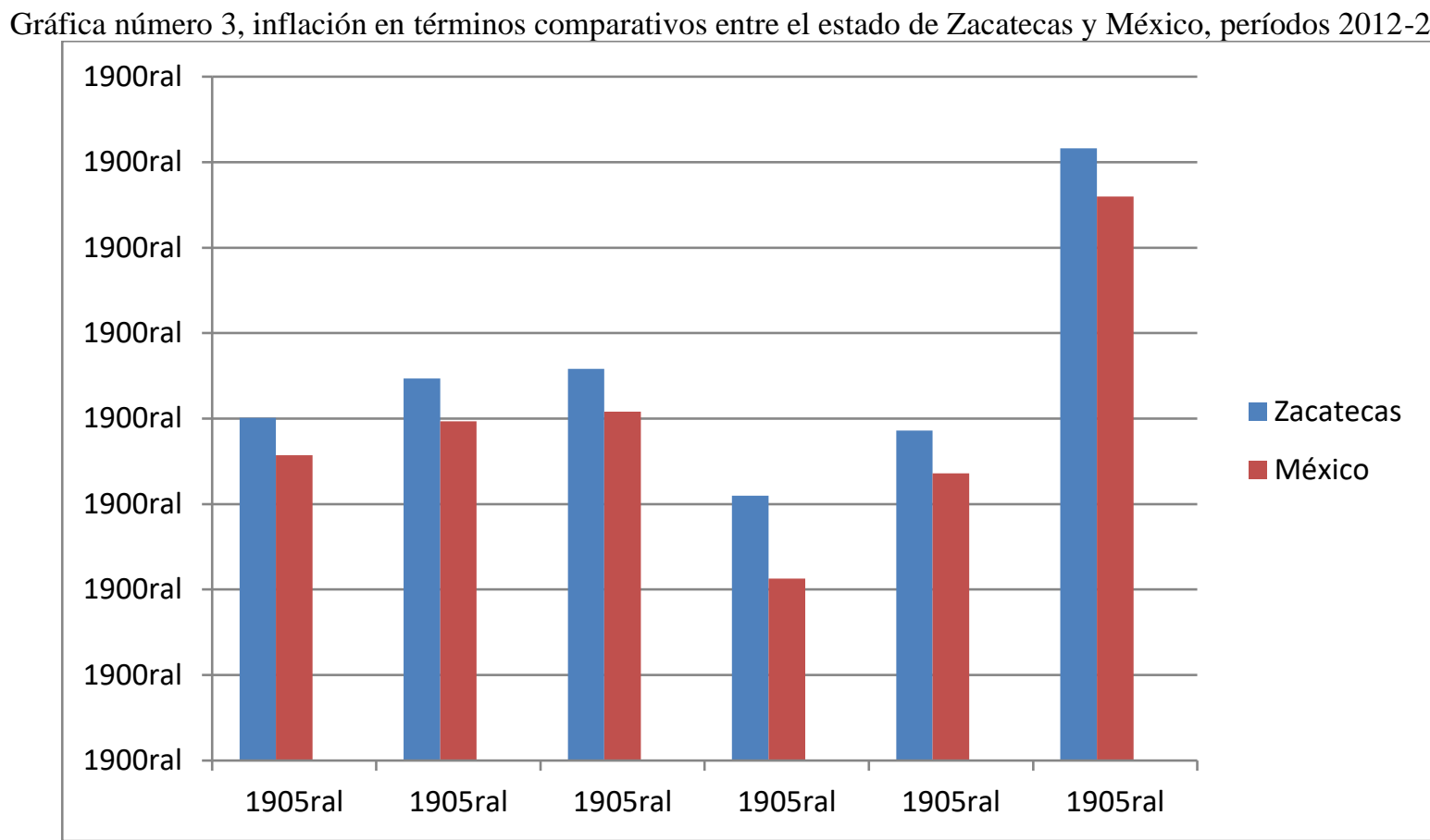

Fuente: Información contenida en el LEA, UAEUAZ, septiembre del 2017.

Como se puede ver en la gráfica número 3, la inflación en el estado de Zacatecas sobrepasa a la existente en México durante los años del 2012 al 2017, un efecto contradictorio dada la condición de pobreza en la cual se encuentra el estado con respecto al resto del país.

Si bien la inflación está en un rango denominado por los órganos oficiales como controlada (por debajo del 10\%), el aumento considerable para el año 2017 tanto para Zacatecas como para México se puede expandir dados los últimos acontecimientos en USA (Texas y Florida), estados que proporcionan al país mexicano el $60 \%$ del consumo diario de hidrocarburos (gasolinas, diesel, kerosina, turbosina y demás) y que sus plantas están siendo afectadas de sobremanera lo que hará subir el precio internacional de los productos con el consiguiente aumento de la inflación en los próximos días.

Es uno de los peligros más latentes que se tiene en México y Zacatecas, por lo regular los aumentos de los hidrocarburos traen una reacción en cadena que afecta siempre a los que menos tienen, el pasaje y la transportación en masa de productos de diversa especia aumentará en los próximos días, el estado de México el día de ayer aumento en dos pesos el transporte urbano y este es un indicador de lo que se viene en el futuro tanto en el estado como en el país. 


\section{CONCLUSIONES}

La inflación repercute de manera directa en las capas poblacionales más vulnerables, las cuales representan más del 65\% de la población en México, éstas sufren de manera directa los aumentos de los precios en los bienes y servicios, los cuales en los últimos años han sido más notorios en los artículos de primera necesidad.

La inflación en México ha sido contralada por las autoridades correspondientes, dando como resultados un crecimiento promedio por debajo del 5\%, aunque es necesario señalar que para el año 2017 no ha tenido un control por parte del gobierno federal al alcanzar un promedio del $6.8 \%$, nivel no alcanzado en años anteriores.

También es necesario adelantar que el cambio de administración federal para el año 2018 fue un detonante muy peligroso en el control absoluto de la inflación.

Entre septiembre de 2016 y el mismo mes de 2017, los precios crecieron 6.66 por ciento, el mayor registro en 16 años. 


\section{BIBLIOGRAFÍA}

Constitución Política de los Estados Unidos Mexicanos, México, 2017.

Instituto Nacional de Estadística Geografía e Informática, varios años, México, 2017.

Laboratorio de Economía Aplicada, Unidad Académica de economía de la Universidad Autónoma de Zacatecas, varios años, México, 2017.

Periódico La Jornada, IsRael RodríGueZ, Martes 4 de julio de 2017, p. 17, México, 2017. León Bendesky, sección Economía, 6-nov-17, México, 2017.

Secretaría de Economía, varios años, México, 2017.

Secretaría de Hacienda y Crédito Público, varios años, México, 2017.

Robles S., Alcances y limitaciones del neoliberalismo en México y Zacatecas, 2000-2014, Ed UAZ, México, 2015

El fracaso del modelo neoliberal en México 2000-2016 ponencia, 4to. Congreso Latinoamericano de Ciencias Sociales, UASC, UAZ, México, junio 2016.

El salario mínimo en México, repercusiones económicas 2016, Ponencia, Congreso Academia Journals, Fresnillo, Zacatecas, México, febrero 2017.

El comportamiento del PIB y el empleo en México 2012-2016 Congreso Internacional de Investigación, Academia Journals, Celaya 2017 